\title{
Thoracic Duct Embolization for Chyle Leakage after Thyroid Surgery
}

\author{
Inhwa Lee ${ }^{1}$, Hyeung Kyoo Kim${ }^{1}$, Jeonghun Lee ${ }^{1}$, Euy Young Soh ${ }^{1}$ \\ and Jinoo $\mathrm{Kim}^{2}$
}

Departments of Thyroid Endocrinology Surgery ${ }^{1}$ and Radiology², Ajou University Medical Center, Ajou University School of Medicine, Suwon, Korea

Chyle leakage (CL) due to lymphatic injuries is one of the rare complications that can develop after thyroidectomy. There are few studies on lymphatic embolization performed in case of $\mathrm{CL}$ after thyroid surgery. We report two cases of $\mathrm{CL}$ after thyroid surgery that were effectively treated by thoracic duct embolization. The patients had previously undergone total thyroidectomy with central compartment neck dissection with or without modified radical neck dissection. The amount of drainage from the operative site was $>1000 \mathrm{~mL}$ per day in one patient and $>500 \mathrm{~mL}$ per day in the other. In both cases, CL stopped after the thoracic duct embolization. Thoracic duct embolization seems to be an effective and important treatment option for $\mathrm{CL}$ after thyroid surgery.

Key Words: Thoracic duct, Chyle, Thyroid gland, Thyroidectomy

\section{Introduction}

Thyroid cancer is presently the most common type of cancer in Republic of Korea." The primary treatment of thyroid cancer is surgery. ${ }^{2)}$ Chyle leakage (CL) due to lymphatic injuries is one of the rare complications that can develop after thyroidectomy, with a reported incidence of 0.5-1.4\% after thyroidectomy and 2-8\% after neck dissections. ${ }^{3)}$ There are various options to treat $\mathrm{CL}$ such as low-fat diet, total parenteral nutrition (TPN), somatostatin administration, lymphatic embolization, and surgery.

There are some reports on lymphatic embolization performed in case of $\mathrm{CL}$ after abdominal or pelvic surgery. ${ }^{4,5}$ However, there are few studies on lymphatic embolization performed in case of $C L$ after thyroid surgery.

This report describes two cases of $C L$ developing after thyroid surgery that were effectively treated by thoracic duct embolization.

\section{Case Reports}

\section{Case 1}

A 41-year-old man had been diagnosed with papillary thyroid cancer $(1.6 \mathrm{~cm})$ in the left thyroid lobe during his regular medical checkup. There was no past history except old pulmonary tuberculosis. Several left level 3 metastatic lymph nodes were confirmed by preoperative ultrasonography and fine-needle aspiration biopsy. The patient underwent total thyroidectomy with central compartment neck dissection and left modified radical neck dissection. On the postoperative day (POD) 1, $1170 \mathrm{~mL}$ was drained through the operative site drainage tube, and $1157 \mathrm{~mL}$ was drained on POD 2. During this time, TPN was administered. On

Received December 3, 2019 / Revised December 3, 2019 / Accepted March 23, 2020

Correspondence: Jinoo Kim, MD, Department of Radiology, Ajou University Medical Center, 164 World Cup-ro, Yeongtong-gu, Suwon 16499, Korea

Tel: 82-31-219-5829, Fax: 82-31-219-5862, E-mail: jinoomail@gmail.com

Copyright (c) the Korean Thyroid Association. All rights reserved.

(i) (\$) This is an open-access article distributed under the terms of the Creative Commons Attribution Non-Commercial License (http://creative(c) (1) \& commons.org/licenses/by-nc/4.0/), which permits unrestricted non-commercial use, distribution, and reproduction in any medium, provided the original work is properly cited. 
POD 3, interventional radiology was consulted for thoracic duct embolization. After embolization, on POD 4, the amount of drainage sharply decreased to $18 \mathrm{~mL}$ per day, after which the patient was discharged without any problems on POD 6 (Table 1).

\section{Case 2}

A 49-year-old woman had been diagnosed with papillary thyroid cancer $(1.8 \mathrm{~cm})$ on the thyroid isthmus by ultrasonography performed due to change in voice. There was no past history except hemorrhoidectomy. She underwent total thyroidectomy with central compartment neck dissection. On PODs 1, 2, 3, and 4, $125 \mathrm{~mL}, 101 \mathrm{~mL}, 186 \mathrm{~mL}$, and $132 \mathrm{~mL}$, respectively, were drained through the operative site drainage tube. During this time, TPN was administered. On POD 5, the amount of CL increased, $639 \mathrm{~mL}$ was drained, and we started to administer somatostatin. On PODs 6 and 7, $485 \mathrm{~mL}$ and $563 \mathrm{~mL}$, respectively, were drained. On POD 8, the patient was referred to interventional radiology for thoracic duct embolization. After the procedure, the drainage tube was accidentally removed, but there was no swelling at the operative site after embolization. The patient started diet and was discharged without any problems on POD 10 (Table 1).

\section{Technique}

We performed thoracic duct embolization with the following technique. The patient lay on the fluoroscopy table in supine position during the procedure. A highfrequency $(7-15 \mathrm{MHz})$ linear transducer $\left(\mathrm{HD} 11 \mathrm{XE}{ }^{\mathbb{R}}\right.$; Philips Medical Solutions, Mountain View, CA, USA) was used to guide a $25-G$ fine needle into a lymph node located in the upper thigh (Fig. 1). Lipiodol (Guerbet, Aulnay-sous-Bois, France) was manually injected at a rate of $1 \mathrm{~mL} / \mathrm{min}$ under fluoroscopic and/or C-arm computed tomography guidance (DynaCT; Siemens, Erlangen, Germany). Intranodal Lipiodol lymphangiography was performed until the Lipiodol penetrated the retroperitoneal lymphatic vessels, passed through the cisterna chyli, and finally reached the thoracic duct to reveal the leakage site. Extravasation of Lipiodol was identified at the upper thoracic level where the extravasated Lipiodol was evacuated through the operative site drain (Fig. 2). Transabdominal puncture of the cisterna chyli was performed using a 22-G needle under fluoroscopic guidance (Fig. 3). A 2.0-Fr microcatheter was co-axially advanced into the thoracic duct. A detachable coil and glue mixture (N-butyl cyanoacrylate [B. Braun Melsungen AG,

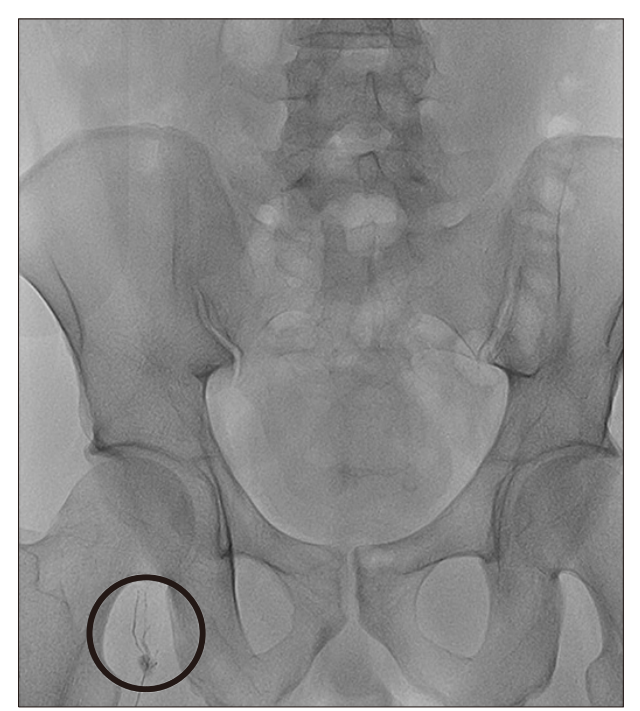

Fig. 1. A single lymph node in the right thigh was punctured using a $25-G$ needle.

Table 1. Characteristics of patients with chyle leakage

\begin{tabular}{cccccccc}
\hline Case Age/Sex & Surgery & $\begin{array}{c}\text { Histologic } \\
\text { type }\end{array}$ & $\begin{array}{c}\text { Amount of drain } \\
\text { before the TDE } \\
(\mathrm{mL} / \text { day })\end{array}$ & $\begin{array}{c}\text { The day of } \\
\text { the TDE }\end{array}$ & $\begin{array}{c}\text { Amount of drain } \\
\text { after the TDE } \\
(\mathrm{mL} / \text { day })\end{array}$ & $\begin{array}{c}\text { The day of } \\
\text { discharge }\end{array}$ \\
\hline 1 & $41 / \mathrm{M}$ & $\begin{array}{c}\text { TT }+ \text { CCND } \\
\text { Lt. mRND }\end{array}$ & PTC & 1157 & POD 3 & 18 & POD 6 \\
2 & 49/F & TT + CCND & PTC & 563 & POD 8 & $(-)^{*}$ & POD 10 \\
\hline
\end{tabular}

*The drainage tube was accidentally removed.

CCND: central compartment neck dissection, F: female, Lt.: left, M: male, mRND: modified radical neck dissection, POD: postoperative day, PTC: papillary thyroid carcinoma, TDE: thoracic duct embolization, TT: total thyroidectomy 


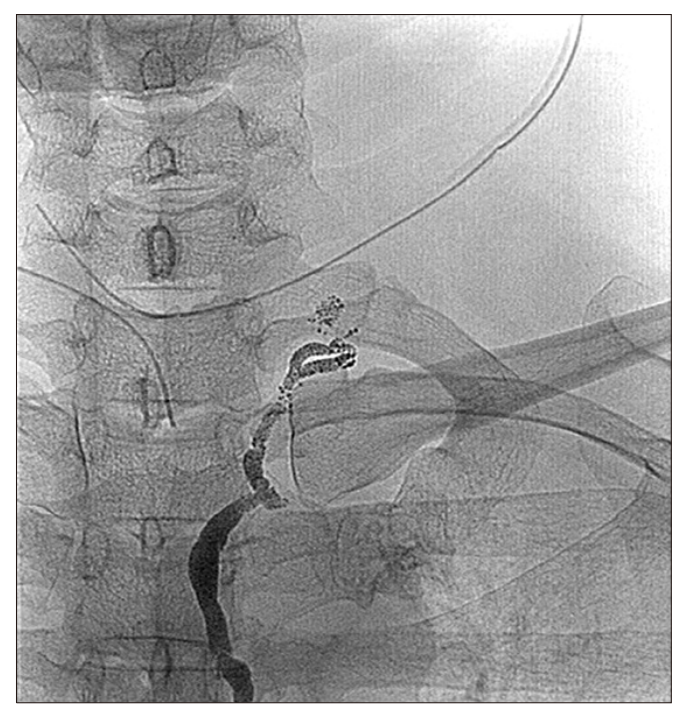

Fig. 2. The leakage site was detected near the cervical portion of the thoracic duct.

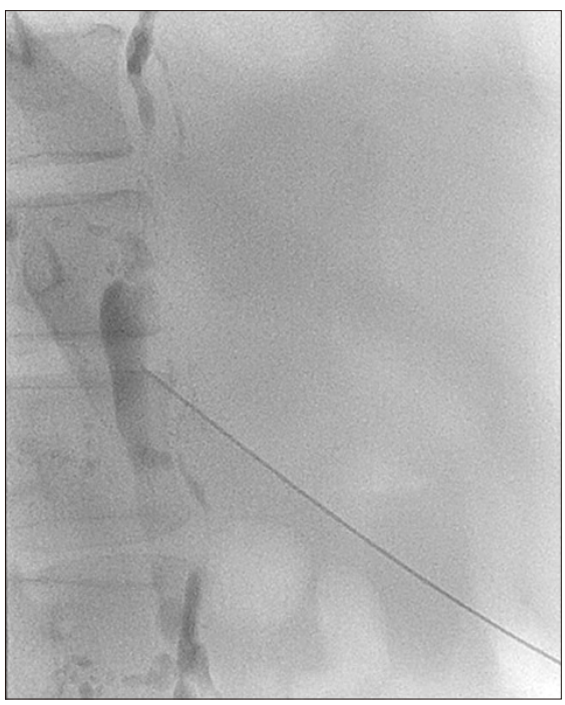

Fig. 3. The cisterna chyli was accessed using a 22-G needle, followed by co-axial delivery of a 2.0-Fr microcatheter into the thoracic duct.

Melsungen, Germany] diluted in Lipiodol at a ratio of 1:1) were used to embolize the distal thoracic duct just below the leakage site (Fig. 4). On the day after the procedure, follow-up chest $\mathrm{X}$-ray was performed (Fig. 5). The study protocol was approved by the Institutional Review Board of Ajou Medical Center (AJIRB-MEDMDB-19-090). Informed consent was not obtained because of the retrospective chart review study design and use of only deidentified clinicopathological information.

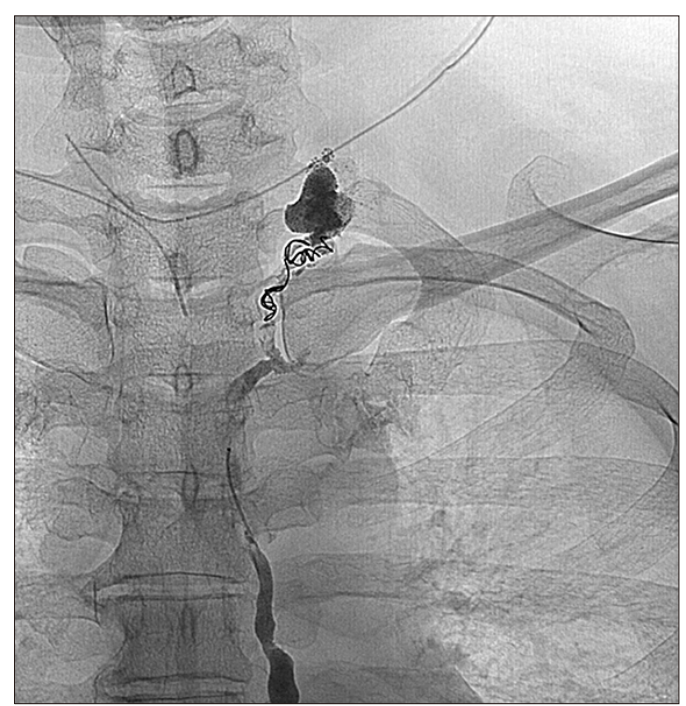

Fig. 4. Coil and glue were used to embolize the distal thoracic duct just below site of leakage.

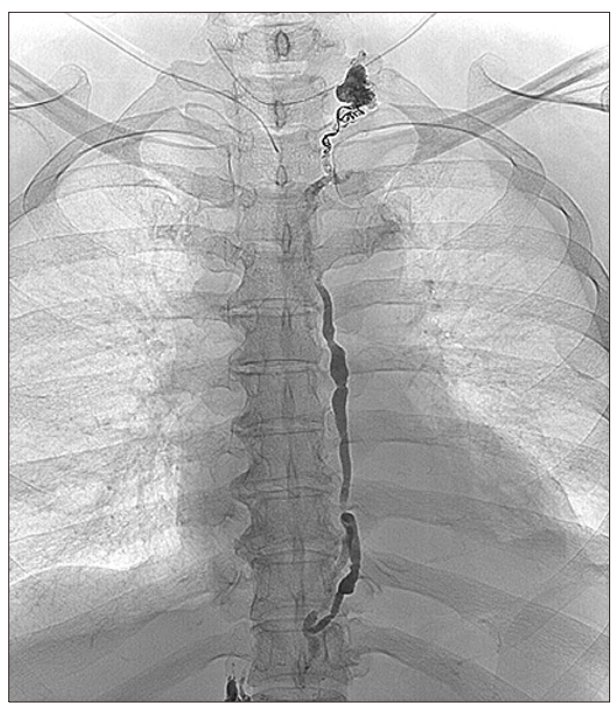

Fig. 5. Post-embolization state.

\section{Discussion}

The thoracic duct originates from the cisterna chyli. Within the abdomen, the thoracic duct ascends along the anterior surface of the lumbar vertebra. It continues to ascend through the thorax, entering the neck. At the neck, as the most common anatomy, it turns inferiorly and terminates at $1 \mathrm{~cm}$ of the confluence of the left internal jugular vein (IJV) and left subclavian vein. ${ }^{3}$ The thoracic duct is lined by of a thin wall of cloudy, white color. It is the largest lymphatic 
vessel in the body and the main trajectory of the lower and upper left body. All the body's lymphatic fluid, except that in the upper right body, enters the thoracic duct.

Unintentional damage to the thoracic duct can occur during neck surgery. There are several causes of thoracic duct injury: proximity to the IJV, thin vessel wall, ${ }^{6)}$ prior irradiation, ${ }^{7)}$ and presence of metastatic lesions at the confluence of the IJV and subclavian vein. ${ }^{8)}$

$\mathrm{CL}$ can cause various problems. Delayed wound healing, infection, or wound breakdown with fistula formation can result from $\mathrm{CL}^{3{ }^{3)}}$ Dehydration, electroIyte disturbances, malnutrition, and immunosuppression can also be caused by $C L .{ }^{6,9)} \mathrm{CL}$ can result in chylothorax, which presents clinically with shortness of breath, tachypnea, and chest pain. ${ }^{3)}$

Treatment of $\mathrm{CL}$ includes conservative measures such as hydration, low-fat diet, TPN, pressure dressings, suction drainage, and somatostatin administration in case of low-output $\mathrm{CL}(<500 \mathrm{~mL} / \text { day })^{3)}$ The patient is usually required to tolerate a long hospitalization period during this time. High-output $\mathrm{CL}(>500 \mathrm{~mL} /$ day) often responds unsatisfactorily to conservative management alone and requires surgical intervention. However, with surgical re-exploration, local inflammation from extravasated chyle can make thoracic duct identification difficult. ${ }^{3)}$ Furthermore, the patient has to endure the burden of reoperation. Among the various options, lymphatic embolization possesses an advantage, owing to its minimally invasive nature. Because the treatment effect appears almost immediately, prolonged hospitalization is not required. The complications from reoperation can also be avoided. Chen and Itkin reported that the overall success rate of thoracic duct embolization in traumatic chylothorax was $91 \% .{ }^{10}$ However, little is known about the success rate of thoracic duct embolization in cases of $\mathrm{CL}$ after thyroid surgery.

In our two cases, $\mathrm{CL}$ stopped after the procedure. The patients returned to normal diet and were discharged without any problems. In both cases, thoracic duct embolization was an effective treatment option.

Although more studies on thoracic duct embolization for $\mathrm{CL}$ after thyroid surgery are needed, it seems to be an effective treatment option for $C L$ after thyroid surgery.

\section{Conflicts of Interest}

No potential conflict of interest relevant to this article was reported.

\section{Orcid}

Inhwa Lee: https://orcid.org/0000-0002-4254-6541 Hyeung Kyoo Kim: https://orcid.org/0000-0003-2165-2334 Jeonghun Lee: https://orcid.org/0000-0003-4925-9104 Euy Young Soh: https://orcid.org/0000-0002-8483-6416 Jinoo Kim: https://orcid.org/0000-0001-7238-2528

\section{References}

1) Ahn HS, Kim HJ, Welch HG. Korea's thyroid-cancer "epidemic"--screening and overdiagnosis. N Engl J Med 2014; 371(19):1765-7.

2) Haugen BR, Alexander EK, Bible KC, Doherty GM, Mandel SJ, Nikiforov YE, et al. 2015 American Thyroid Association management guidelines for adult patients with thyroid nodules and differentiated thyroid cancer: The American Thyroid Association guidelines task force on thyroid nodules and differentiated thyroid cancer. Thyroid 2016;26(1):1-133.

3) Delaney SW, Shi H, Shokrani A, Sinha UK. Management of chyle leak after head and neck surgery: review of current treatment strategies. Int J Otolaryngol 2017;2017:8362874.

4) Baek Y, Won JH, Chang SJ, Ryu HS, Song SY, Yim B, et al. Lymphatic embolization for the treatment of pelvic lymphoceles: preliminary experience in five patients. J Vasc Interv Radiol 2016;27(8):1170-6.

5) Nadolski GJ, Chauhan NR, Itkin M. Lymphangiography and lymphatic embolization for the treatment of refractory chylous ascites. Cardiovasc Intervent Radiol 2018;41(3):415-23.

6) Crumley RL, Smith JD. Postoperative chylous fistula prevention and management. Laryngoscope 1976;86(6):804-13.

7) Swanson MS, Hudson RL, Bhandari N, Sinha UK, Maceri $\mathrm{DR}$, Kokot N. Use of octreotide for the management of chyle fistula following neck dissection. JAMA Otolaryngol Head Neck Surg 2015;141(8):723-7.

8) Ahn D, Sohn JH, Jeong JY. Chyle fistula after neck dissection: an 8-year, single-center, prospective study of incidence, clinical features, and treatment. Ann Surg Oncol 2015;22 Suppl 3:S1000-6.

9) Ilczyszyn A, Ridha H, Durrani AJ. Management of chyle leak post neck dissection: a case report and literature review. J Plast Reconstr Aesthet Surg 2011;64(9):e223-30.

10) Chen E, Itkin M. Thoracic duct embolization for chylous leaks. Semin Intervent Radiol 2011;28(1):63-74. 